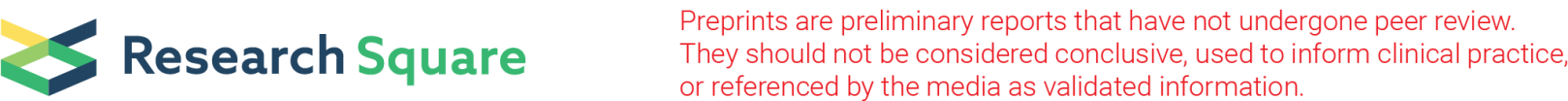

\section{Relationships among Neutrophil-to-Lymphocyte Ratio, Serum Albumin Level, and Platelet-to- Lymphocyte Ratio with In-stent Restenosis in Cerebrovascular Bare-Metal Stenting}

\section{Zhuoxi Xie}

Affiliated Hospital of Guilin Medical University

Jiajun Wei

Affiliated Hospiital of Guilin Medical University

Ligen Fan

Affiliated Hospital of Guilin Medical University

Fan Yang

Affiliated Hospital of Guilin Medical University

Qiang Fang

Affiliated Hospital of Guilin Medical University

Zhouming Zhu

Affiliated Hospital of Guilin Medical University

Jing Wu

Affiliated Hospital of Guilin Medical University

Hao Li ( $\nabla$ lihao_glmc@163.com )

Guilin Medical University Affiliated Hospital

\section{Research}

Keywords: in-stent restenosis, cerebrovascular bare-metal stenting, neutrophil-to-lymphocyte ratio, serum albumin, platelet-to-lymphocyte ratio

Posted Date: January 28th, 2021

DOI: https://doi.org/10.21203/rs.3.rs-154403/v1

License: (1) This work is licensed under a Creative Commons Attribution 4.0 International License. Read Full License 


\section{Abstract}

Background: In-stent restenosis (ISR) remains a serious clinical problem that affects the efficacy of endovascular treatment. Inflammation and platelet activation play an important role in the development of ISR after cerebrovascular bare-metal stenting. The neutrophil-to-lymphocyte ratio (NLR) and platelet-tolymphocyte ratio (PLR), which are the indicators of inflammation, have been associated with stent restenosis, while low serum albumin (ALB) level has been associated with the adverse outcomes of cardiovascular diseases. We therefore explored the associations among preprocedural NLR, ALB level, and PLR with ISR after cerebrovascular bare-metal stenting and then investigated the value of preprocedural NLR, ALB level, and PLR for the development of ISR.

Methods: In this study, we enrolled 223 patients who underwent cerebrovascular bare-metal stenting (both intracranial and extracranial) and retrospectively analyzed their clinical, hematological, and angiographic data. Radiographic evaluations were performed at 6 and 12 months after the procedure.

Results: ISR was defined as $\geq 50 \%$ stenosis of the treated lesion. Patients were divided into two groups based on the occurrence of ISR. ISR occurred in 89 (39.91\%) of the 223 included patients. Increased preprocedural NLR $(p<0.001)$ and PLR $(p<0.001)$ and decreased pre-procedural ALB level $(p<0.001)$ were significantly associated with ISR.

Conclusions: High pre-procedural NLR and PLR, and low pre-procedural ALB level may be used as predictors of ISR after cerebrovascular bare-metal stenting.

Trial Registration: retrospective registration.

\section{Introduction}

Stroke is the leading cause of death and disability among Chinese adults ${ }^{1}$. About 3 million people suffer a stroke every year in China. Notably cerebrovascular atherosclerotic stenosis is the main cause of ischemic stroke, and it is associated with a high risk of stroke recurrence ${ }^{2}$. Presently, the clinical treatment methods available for cerebral atherosclerotic stenosis include drugs (e.g. non-steroidal antiinflammatory drugs [NSAIDs]), endovascular treatment, and surgical treatment. Endovascular treatment has become a common treatment option for cerebrovascular stenosis owing to its effectiveness and the minimal invasive nature ${ }^{3}$. Endovascular treatment can be classified into 3 subtypes: simple balloon dilatation angioplasty (BDA), bulb-expanding stent or self-expanding stent(whose efficacy is comparable to that of BDA), and stent implantation(which has the highest effectiveness among the 3 subtypes) ${ }^{4}$. However, the occurrence of in-stent restenosis (ISR) after stent placement can render the stent ineffective, which is associated with a high risk of recurrent cerebrovascular accidents ${ }^{5}$. Therefore, the identification of simple and effective indicators that can predict the occurrence of ISR is critical to facilitate the timely initiations of preventive treatment in order to reduce the incidence rate of ISR. 
The pathophysiological mechanism of ISR has not been fully elucidated until date, and inflammatory response and platelet activation are generally considered to be the chief causes of ISR. The conventional indicators, such as hypersensitive C-reactive protein ( $\mathrm{h}-\mathrm{CRP})^{6}$, procalcitonin (PCT) levels ${ }^{7}$, and the mean platelet volume (MPV) ${ }^{8}$, have been proven to be correlated with ISR. However, the conventional indicators have low specificity and present with limitations in clinical prediction of ISR. The neutrophil-tolymphocyte ratio (NLR) is a sensitive indicator of inflammation, and has been confirmed to be associated with ISR after implantations of carotid artery stent ${ }^{9}$, although its relationship with the other types of cerebrovascular ISR remains to be clarified. Serum albumin (ALB) is a negative acute-phase inflammatory protein. Its concentration decreases during the inflammatory reaction phase. The decrease in the ALB level is related to an increase in the blood viscosity ${ }^{10}$. The platelet-to-lymphocyte ratio (PLR) is a sensitive indicator that reflects platelet activation and inflammation. Past studies have confirmed that ALB and

PLR are associated with coronary artery stent restenosis ${ }^{11,12}$, however, their relationship with restenosis in cerebrovascular bare-metal stents remains unclear. The present study aimed to explore the relationships between the preoperative peripheral blood ALB level and PLR with cerebrovascular bare-metal restenosis as well as to compare the predictive efficacies of NLR, ALB, and PLR for intracranial restenosis of cerebrovascular bare-metal stents in order to establish a strong predictor of ISR for clinical reference in the future.

\section{Methods}

\subsection{Study Population}

We retrospectively evaluated the medical records of 223 patients who underwent elective implantation of cerebrovascular stents (both intracranial + extracranial) at the Department of Neurology between January 2018 and December 2020. The information on patients' sex, age, body mass index (BMI), smoking status, the presence of hypertension/diabetes/coronary heart disease, lipid profile, stent placement location, stent type, and other characteristics were retrieved from the medical records. The patients were followedup for 6-12 months using computed tomography angiography (CTA) or digital subtraction angiography (DSA) and, based on the results into the ISR and no ISR(NO-ISR) groups. The patient inclusion criteria included the following: the implantation of intracerebral vascular stent for the dilation of original stenosis of $>70 \%$ at our hospital and a follow-up study at our hospital within 6-12 months involving examinations such as hematology, CTA, or DSA. The patient exclusion criteria included the following: (1) the lack of relevant baseline data; (2) receipt of irregular anti-platelet therapy after surgery; and the presence of (3) acute and chronic infection; (4) severe liver and kidney diseases; (5) blood diseases or benign and malignant tumors; (6) iodine allergy; (7) a recent history of major trauma and surgery; (8) heart failure; (9) systemic immune diseases and the use of inflammation inhibitors; and (10) pregnancy. The smoking habit referred to daily smoking since $>6$ months. BMI was defined as weight $(\mathrm{kg})$ divided by height squared $\left(\mathrm{m}^{2}\right)$. The presence of hypertension was defined according to the 2017ACC/AHA diagnostic criteria ${ }^{13}$ or based on prior treatment for hypertension. The presence of diabetes was defined according to the $2019 \mathrm{ESC} / \mathrm{EASD}$ guidelines for diabetes management diagnostic criteria ${ }^{14}$ or prior 
diabetes treatment. Presence of dyslipidemia was defined according to the 2019ESC/EAS guidelines for the management of dyslipidemia ${ }^{15}$ or based on prior treatment for dyslipidemia.

\subsection{Laboratory Assays}

The demographic characteristics and the laboratory and imaging results were retrieved from the medical records. The fasting peripheral blood samples were collected before surgery. EDTA tubes were used for hematological testing and non-anticoagulant tubes were used for biochemical analysis. An automated blood analyzer (Sysmex XE-2100 Kobe, Japan) was used for sample analysis, and the standard laboratory techniques were employed for testing. NLR was defined as the ratio of neutrophil count to the lymphocyte count, and PLR was defined as the ratio of platelet count to the lymphocyte count.

\subsection{Procedures and Evaluation}

All cerebrovascular stent implantations were performed in compliance with the relevant guidelines and recommendations of the hospital. All patients received the following drug treatment in accordance with the guidelines before surgery, aspirin (100-mg/day) and clopidogrel (75-mg/day) for $3-5$ days, statins for lowering the lipid levels, and specific drugs for the management of hypertension and diabetes. If stenting was planned within the subsequent $72 \mathrm{~h}$, an additional $300 \mathrm{mg}$ of clopidogrel was administered $4 \mathrm{~h}$ prior to the surgery. All surgical procedures were performed by qualified doctors. The operation was performed under local or general anesthesia, and $8 \mathrm{~F}$ arterial sheathing was inserted into the femoral artery by the modified Seldinger method; in addition, heparin $(70-\mathrm{IU} / \mathrm{kg})$ was administered intraoperatively, and the catheter was irrigated with continuous high-pressure infusion of heparin saline to reduce the risk of thromboembolic events. After the arrival of $8 \mathrm{~F}$ guiding sheath to the stenosis segment, the stenosis degree was calculated in accordance with the standard North American symptomatic carotid endarterectomy test (NASCET). The selection of appropriate protective device, requirement for preexpansion, and the determination of stent location and size were performed according to the condition of the blood vessel. One of the following bare-metal stents was selected: Wallstent (Boston Scientific, US), Neuroform EZ (US), Enterprise (Codman, US), Express SD/LD(Boston Scientific, US), Apollo (Shanghai Minimally Invasive Medical Device Co., Ltd. ), Wingspan (Boston Scientific). A residual stenosis rate of $\leq$ $30 \%$ indicated that the angiography was successful. If the residual stenosis rate was $>50 \%$, it was usually expanded after angiography, and, if thromboembolism was not detected, the protective device was recovered. After 3-6 months of postoperative administration of aspirin and clopidogrel, patients with clopidogrel resistance were prescribed a single anti-platelet agent for life (usually aspirin). Patients with clopidogrel resistance should be prescribed ticagrelor or other anti-platelet agents, and statins for longterm lipid regulation. The patients were followed up for 6-12 months, and CTA or DSA were rechecked to assess the occurrence of ISR. ISR was defined as $>50 \%$ stenosis within the stent or within 3-mm of the edge of the stent, as confirmed by angiography ${ }^{5}$.

\subsection{Statistical Analyses}

All data analyses were performed using the SPSS version 18.0. Categorical variables are expressed in numbers and percentages, and continuous variables were expressed as mean \pm standard deviation or as 
the median and quartile range. Quantitative data were expressed as ( $X \pm S$ ), and t-test was performed to compare the mean values between the two groups; the count data was expressed by ratio (n, \%), and the $\chi^{2}$ test was used for comparison. Multivariate logistic regression analysis was performed to determine the independent predictor of ISR. Receiver operator characteristic curves (ROCs) of NLR, ALB, and PLR for the prediction of ISR were used to calculate the cut-off value, area under the curve (AUC), sensitivity, and specificity of each indicator, while AUC was used as the indicator of performance. $p<0.05$ was considered to be statistically significant.

\section{Results}

The baseline demographic, clinical, and procedural characteristics of the patients are depicted in Table 1. The mean age of the patients was $62 \pm 9$ years, and the subjects included $172(77.13 \%)$ male patients. Patients were categorized into two groups, namely ISR and non-ISR (NO-ISR) groups. ISR occurred in $39.91 \%(n=89)$ of the patients.

Patients in the ISR group were older than those in the NO-ISR group. The ISR group showed a significantly higher prevalence rate of diabetes and significantly lower BMI than did the NO-ISR group. No significant differences were noted between the two groups with respect to age, sex, smoking status, incidence rates of hypertension and hyperlipidemia, and stent location, length, and diameter.

Table 2 lists the laboratory findings of the study population at the baseline. Patients with ISR had a higher baseline value of NLR $(p<0.001)$, and PLR $(p<0.001)$ and lower values of ALB $(p<0.001)$ and lymphocyte count $(p=0.003)$ than did those without ISR. As shown in Table 2 and Fig. 1, patients with ISR showed significantly lower ALB levels than did patients without ISR $(39.43 \pm 2.96$ vs $42.13 \pm 2.77 \mathrm{~g} / \mathrm{L}$, $p<0.001)$. In contrast, ISR patients showed higher NLR and PLR values than those without ISR (3.19 \pm 1.49 vs $2.46 \pm 1.12 ; p<0.001,148.60 \pm 33.73$ vs $125.36 \pm 34.88, p<0.001)$.

The ROC curve analysis was employed to calculate the truncation values AUCs, sensitivities, specificities, and $95 \%$ confidence intervals (Cls) of NLR, ALB, and PLR (Table 3), and the ROC curves of NLR, ALB, and PLR were are depicted in Fig. 2. Multivariate logistic regression analysis revealed that the BMI (odds ratio [OR], 1.204; 95\% confidence interval [Cl], 1.091-1.327, $p<0.001)$ and preoperative ALB level (OR, 0.685; $95 \% \mathrm{Cl}, 0.611-0.769, p<0.001)$, NLR (OR, 1.323; 95\% Cl, 1.056-1.658, $p=0.015)$, and PLR (OR, 1.019; $95 \% \mathrm{Cl}, 1.009-1.028, p<0.001$ ) were independent risk factors for ISR (Table 4).

\section{Discussion}

In this study, we analyzed the predictive value of preoperative peripheral blood NLR and PLR counts and the ALB level for ISR in patients who underwent cerebrovascular bare-metal stent implantation. We noted that higher preoperative NLR (>2.28), PLR (>140.25), and lower ALB level ( $\leq 42.32)$ were associated with the development of ISR, and hence these parameters could help predict the occurrence of ISR. The incidence rate of ISR after cerebrovascular stenting was recorded to be $20-30 \%{ }^{16}$, and the ISR rate was 
found to vary according to the clinical centers and locations. The occurrence of ISR increases the risk of cerebrovascular accidents, increase costs, and reduces the quality of life ${ }^{17}$. Therefore, the etiology of stent restenosis has become a research hotspot. At present, the pathophysiological mechanism of ISR is considered to mainly involve: immune inflammatory response, vascular remodeling, new atherosclerosis, and thrombosis, among others. ${ }^{18-20}$

The relationship between NLR, as a sensitive indicator of inflammation, and ISR was confirmed in previous studies. NLR has been suggested as a predictor of cerebrovascular ISR ${ }^{9}$ and coronary ISR ${ }^{21}$. Mechanical damage and blood flow changes during stent placement leads to the deposition of platelets and fibrin ${ }^{22}$. The interaction between white blood cells and platelets leads to the recruitment of white blood cells, including neutrophils, into the scaffold segment, which in turn triggers an inflammatory response, in which the involved inflammatory factors lead to the formation of neointima. The neointima is dysfunctional and does not inhibit platelet aggregation. Some subtypes of lymphocytes, such as regulatory T-cells (Tregs), suppress autoimmune inflammation and maintain homeostasis ${ }^{23}$. Therefore, increased NLR counts indicate an active inflammatory response and promotes the occurrence of ISR.

PLR is a new indicator that reflects platelet activation and inflammation status, and it is widely used to predict adverse outcomes of cardiovascular and cerebrovascular disease, such as stroke ${ }^{24}$, myocardial infarction ${ }^{25}$, and heart failure ${ }^{26}$. S Y et al. confirmed that high PLR is associated with the incidence of coronary ISR ${ }^{12}$. The possible mechanism may be as follows: inflammatory mediators stimulate megakaryocytes to proliferate and produce relatively greater higher number of platelets, which play an important role in the pathogenesis of atherosclerosis ${ }^{27}$. Platelets participate in both the formation of plaques and the formation of fibrin plugs as a result of the rupture of unstable plaques. They can also release thrombin and other substances, thus exacerbating the inflammatory response ${ }^{28}$. Thus, increased platelet count increases the risk of thrombosis, while lymphocytes achieve an inflammation-antiinflammatory balance by suppressing their own inflammatory response ${ }^{23}$. Therefore, inflammation and high platelet activation increases with an increase in the PLR count.

The two new types of inflammatory indicators PLR and NLR are closely related to the progression of atherosclerosis ${ }^{29}$. Shah et al ${ }^{30}$ found that low lymphocyte count is an independent risk factor for coronary heart disease, which will not be affected by the absolute counts of blood cells under different physiological conditions, such as dehydration or exercise. Thus, they may have good predictive value for ISR.

ALB exerts physiological effects such as the regulation of inflammation, anti-oxidation, anti-coagulation, and inhibition of platelet aggregation, which has been confirmed to be related to stroke, cardiovascular disease, and coronary ISR ${ }^{11}, 31$. It can be reversibly combined with nitric oxide (NO) and prostaglandin through oxidation and glycosylation in order to achieve anti-oxidation and regulation of inflammation ${ }^{32}$. ALB also possesses heparin-like activity, probably due to the similarity in their structures and electrostatic charges $^{33}$. M P et al. compared the low ALB group, physiological albumin group, and high ALB group 
using platelet function analyzer and found that the low ALB group was more likely to demonstrate thrombosis ${ }^{34}$. Therefore, hypoalbuminemia can aggravate inflammation, promote platelet aggregation, and aggravate the occurrence of ISR.

Based on our analysis, the most important method that helps reduce the incidence of ISR is monitoring and prevention, which makes standardization of long-term medication and regular review essential. Whole blood cell test is a non-invasive, economical, and simple test method. The identification of blood markers can help predict ISR to facilitate effective patient follow-up study. Once ISR is detected, active intervention is needed to reduce the occurrence of cerebrovascular events. Balloon dilatation or repeated stenting should be considered when anti-platelet drugs or statins are ineffective $\mathrm{e}^{35}$. There continues to be a risk of ISR after repeated stent placement. Because of the high mutation rate of anti-platelet drug resistance genes in the Chinese population, conducting genetic screening for drug resistance, if the conditions permits, is recommended for guide drug selection ${ }^{36}$.

\section{Limitation}

This study has some limitations. First of all, this is a single-center retrospective study that can explain the relationship between clinical factors and ISR. Second, although we included data on laboratory examination and imaging, we could not use intravascular ultrasound or optical coherence tomography or stent restenosis plaque biopsy for further examination. These methods are more quantitative and informative than visual estimation. Finally, we did not conduct a long-term follow-up. Thus, large-scale, prospective, and randomized clinical trials are warranted in the future to assess the clinical applicability of our findings.

\section{Conclusion}

In conclusion, this study demonstrates that diabetes, high BMI, NLR and PLR counts, and low ALB level and lymphocyte count are associated with the high risk of ISR after implantation of cerebrovascular baremetal stents. Thus, the count of NLR and PLR and the value of BMI increase before surgery, while ALB value decline. These factors can act as independent risk factor for ISR in patients implanted with cerebrovascular bare-metal stents. In summary, NLR $>2.28$, ALB level $\leq 42.32$, and PLR $>140.25$ are powerful indicators that independently predict ISR in patients implanted with cerebrovascular bare-metal stents.

\section{Abbreviations}

ALB: serum albumin. NLR: neutrophil to lymphocyte ratio. PLR: platelet to lymphocyte ratio. BMl: the body mass index. ISR: in-stent restenosis. NO-ISR: no in-stent restenosis. CTA: computed tomography angiography. DSA: digital subtraction angiography. DM: diabetes mellitus. ROC: receiver operator characteristic curves. AUC: area under the curve. OR: odds ratio. Cl: confidence interval. 


\section{Declarations}

\section{Ethics approval and consent to participate}

This study was approved by the ethical review committee of the Affiliated Hospital of Guilin Medical University, and written informed consents were obtained from all patients.

\section{Consent for publication}

The authors declared no potential conflicts of interest with respect to the research, authorship, and/or publication of this article.

\section{Availability of data and materials}

The data and material that support the findings of this study are available in the hospital information system of our hospital.

\section{Competing interests}

Not applicable.

\section{Funding}

This study was supported by the Scientific Research and Technology Development Program of Guilin City(NO.2017010911).

\section{Authors' contributions}

All authors contributed to the conception and design, acquisition of data or analysis, interpretation of data, in drafting the article or revising it critically for important intellectual content, and for final approval of the version to be published.

\section{Acknowledgements}

Not applicable.

\section{References}


1. GBD 2016 Neurology Collaborators, Global, regional, and national burden of neurological disorders, 1990-2016: a systematic analysis for the Global Burden of Disease Study 2016.[J].Lancet Neurol, 2019, 18: 459-480.

2. Zuo F T, Liu H, Wu HJ et al. The effectiveness and safety of dual antiplatelet therapy in ischemic cerebrovascular disease with intracranial and extracranial arteriostenosis in Chinese patients: A randomized and controlled trail.[J].Medicine (Baltimore), 2017, 96: e5497-5503.

3. Eckstein H-H, European Society for Vascular Surgery Guidelines on the Management of Atherosclerotic Carotid and Vertebral Artery Disease.[J].Eur J Vasc Endovasc Surg, 2018, 55: 1-2.

4. Qureshi A I, Chaudhry S A, Siddiq F, et al. A randomized trial comparing primary angioplasty versus stent placement for symptomatic intracranial stenosis.[J]. Journal of Vascular \& Interventional Neurology, 2013, 6(2):34-41.

5. Pleva L, Kukla P, Hlinomaz O. Treatment of Coronary In-Stent Restenosis: A Systematic Review[J]. Journal of Geriatric Cardiology Jgc, 2018, 15(2):173-184.

6. Niccoli G, Dato I, Imaeva A E et al. Association between inflammatory biomarkers and in-stent restenosis tissue features: an Optical Coherence Tomography Study.[J].Eur Heart J Cardiovasc Imaging, 2014, 15: 917-925.

7. Zheng D, Mingyue Z, Wei S et al. The Incidence and Risk Factors of In-Stent Restenosis for Vertebrobasilar Artery Stenting.[J].World Neurosurg, 2018, 110: e937-e941.

8. Karauzum K, Bildirici U, Dervis E et al. Preprocedural Mean Platelet Volume Level Is a Predictor of InStent Restenosis of the Superficial Femoral Artery Stents in Follow-Up.[J].Cardiol Res Pract, 2018, 2018: 4572629.

9. Dai Z Z, Li R R, Zhao N et al. Neutrophil to Lymphocyte Ratio as a Predictor of Restenosis After Angioplasty and Stenting for Asymptomatic Carotid Stenosis.[J].Angiology, 2019, 70: 160-165.

10. Arques S, Serum albumin and cardiovascular diseases: A comprehensive review of the literature]. [J].Ann Cardiol Angeiol (Paris), 2018, 67: 82-90.

11. Celik I E, Yarlioglues M, Kurtul A et al. Preprocedural Albumin Levels and Risk of In-Stent Restenosis After Coronary Stenting With Bare-Metal Stent.[J].Angiology, 2016, 67: 478-483.

12. Yılmaz S, Sen F, Ünal S et al. Usefulness of the platelet-to-lymphocyte ratio in predicting bare-metal stent restenosis.[J].Scand Cardiovasc J, 2015, 49: 39-44.

13. Whelton Paul K, Carey Robert M, Aronow Wilbert S et al. 2017 ACC/AHA/AAPA/ABC/ACPM/AGS/ APhA/ASH/ASPC/NMA/PCNA Guideline for the Prevention, Detection, Evaluation, and Management of High Blood Pressure in Adults: Executive Summary: A Report of the American College of Cardiology/American Heart Association Task Force on Clinical Practice Guidelines.[J].Circulation, 2018, 138: e426-e483.

14. Grant P J, Cosentino F. The 2019 ESC Guidelines on diabetes, pre-diabetes, and cardiovascular diseases developed in collaboration with the EASD[J]. European Heart Journal, 2019, 40(39):32153217. 
15. Mach F, Baigent C, Catapano A L et al. 2019 ESC/EAS Guidelines for the management of dyslipidaemias: lipid modification to reduce cardiovascular risk.[J].Eur Heart J, 2020, 41: 111-188.

16. Her A Y, Shin E S, Current Management of In-Stent Restenosis.[J].Korean Circ J, 2018, 48: 337-349.

17. Werner Martin, Drug eluting stents and modern stent technologies for in-stent restenosis.[J].J Cardiovasc Surg (Torino), 2017 Aug;58(4):497-500.

18. Goel S A, Guo L W, Liu B et al. Mechanisms of post-intervention arterial remodelling.[J].Cardiovasc Res, 2012, 96: 363-371.

19. Kim M S, Dean L S. In-stent restenosis.[J]. Cardiovascular Therapeutics, 2011, 29(3):190-198.

20. Srimurugan B, Sigler M, Sankar N M et al. In-stent restenosis: surgical and histopathological perspective.[J].Asian Cardiovasc Thorac Ann, 2018, 26: 114-119.

21. Turak 0, Ozcan F, Isleyen $A$ et al. Usefulness of the neutrophil-to-lymphocyte ratio to predict baremetal stent restenosis.[J].Am J Cardiol, 2012, 110: 1405-1410.

22. Kim V D H, Gijsen F J H, Andrew N, et al. The effects of stenting on shear stress: relevance to endothelial injury and repair.[J]. Cardiovasc Res, 2013, 99: 269-75.

23. Chapman N M , Chi H. MTOR signaling, Tregs and immune modulation[J]. Immunotherapy, 2014, 6(12):1295-1311.

24. İdil S A, Arıkan C S, Uzunkaya F et al. The correlation of the platelet-to-lymphocyte ratio with the severity of stenosis and stroke in patients with carotid arterial disease.[J].Vascular, 2017, 25: 299306.

25. Vakili H, Shirazi M, Charkhkar M et al. Correlation of platelet-to-lymphocyte ratio and neutrophil-tolymphocyte ratio with thrombolysis in myocardial infarction frame count in ST-segment elevation myocardial infarction.[J].Eur J Clin Invest, 2017, 47: 322-327.

26. Durmus E, Kivrak T, Gerin F et al. Neutrophil-to-Lymphocyte Ratio and Platelet-to-Lymphocyte Ratio are Predictors of Heart Failure.[J].Arq Bras Cardiol, 2015, 105: 606-613.

27. Balta S, Demrkol S, Kucuk U. The platelet lymphocyte ratio may be useful inflammatory indicator in clinical practice[J]. Hemodialysis International, 2013, 17(4):668-669.

28. Ahbap E, Sakaci T , Kara E, et al. Neutrophil-to-lymphocyte ratio and platelet-tolymphocyte ratio in evaluation of inflammation in end-stage renal disease.[J]. Clinical Nephrology, 2015, 85(4):199-208.

29. Condado J F, Junpaparp P, Binongo J N, et al. Neutrophil-lymphocyte ratio (NLR) and plateletlymphocyte ratio (PLR) can risk stratify patients in transcatheter aortic-valve replacement (TAVR)[J]. International Journal of Cardiology, 2016, 223:444-449.

30. Shah A D , Denaxas S, Nicholas 0 , et al. Low eosinophil and low lymphocyte counts and the incidence of 12 cardiovascular diseases: a CALIBER cohort study[J]. Open Heart, 2016, 3(2):e000477.

31. Arques S, Human serum albumin in cardiovascular diseases.[J].Eur J Intern Med, 2018, 52: 8-12.

32. Arroyo V, García-M R, Salvatella X, Human serum albumin, systemic inflammation, and cirrhosis.[J].J Hepatol, 2014, 61: 396-407. 
33. Chien S C , Chen C Y, Lin C F , et al. Critical appraisal of the role of serum albumin in cardiovascular disease[J]. Biomarker Research, 2017, 5(1):31-36.

34. Paar Margret,Rossmann Christine,Nusshold Christoph et al. Anticoagulant action of low, physiologic, and high albumin levels in whole blood.[J].PLoS One, 2017, 12: e0182997.

35. Looser P M , Kim L K , Feldman D N . In-Stent Restenosis: Pathophysiology and Treatment[J]. Current Treatment Options in Cardiovascular Medicine, 2016, 18(2):1-12.

36. Cui G Z, Zhang S Y, Zou J et al. P2Y12 receptor gene polymorphism and the risk of resistance to clopidogrel: A meta-analysis and review of the literature.[J].Adv Clin Exp Med, 2017, 26: 343-349.

\section{Tables}

Due to technical limitations, tables are only available as a download in the Supplemental Files section.

\section{Figures}



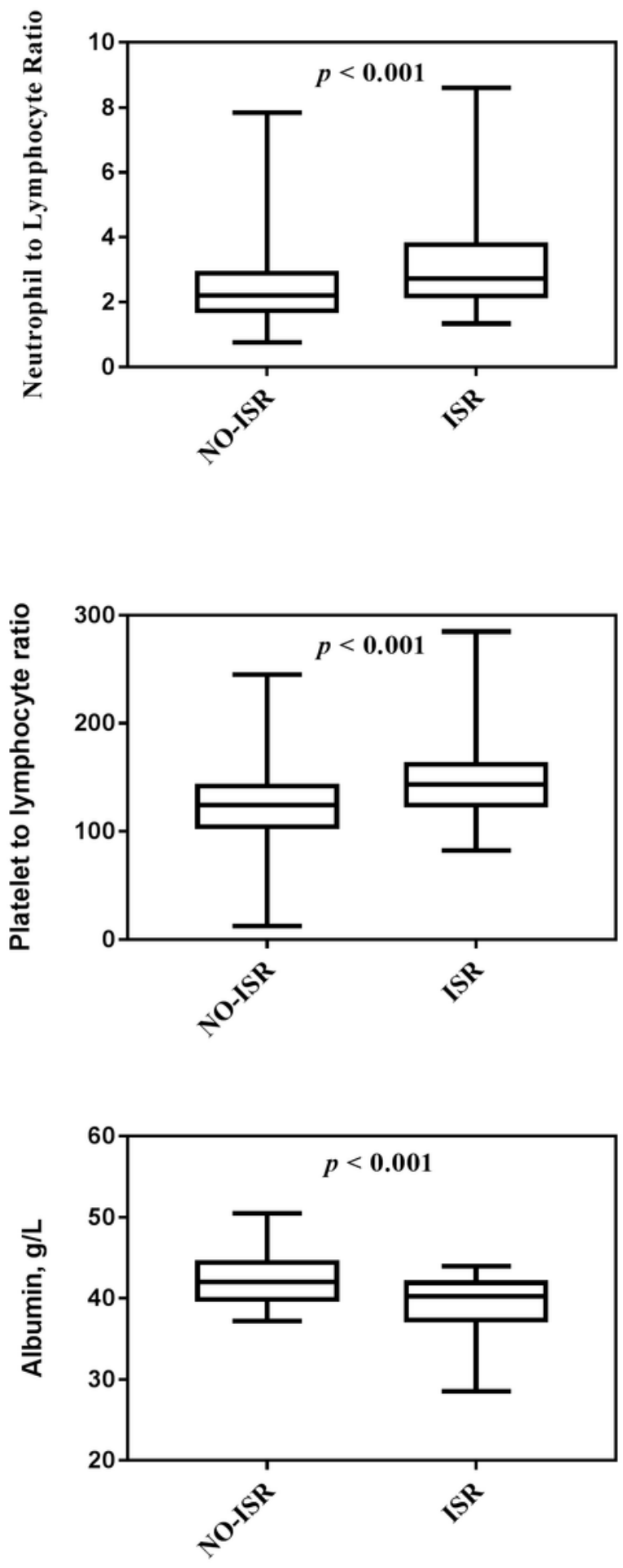

Figure 1

Patients with ISR had a higher baseline value of NLR $(p<0.001)$, and PLR $(p<0.001)$ and lower values of ALB $(p<0.001)$ and lymphocyte count $(p=0.003)$ than did those without ISR. As shown in Table 2 and Figure 1, patients with ISR showed significantly lower ALB levels than did patients without ISR (39.43 \pm 2.96 vs $42.13 \pm 2.77 \mathrm{~g} / \mathrm{L}, \mathrm{p}<0.001)$. In contrast, ISR patients showed higher NLR and PLR values than those without ISR ( $3.19 \pm 1.49$ vs $2.46 \pm 1.12 ; p<0.001,148.60 \pm 33.73$ vs $125.36 \pm 34.88, p<0.001)$. 


\section{ROC Curves for Comparisons}
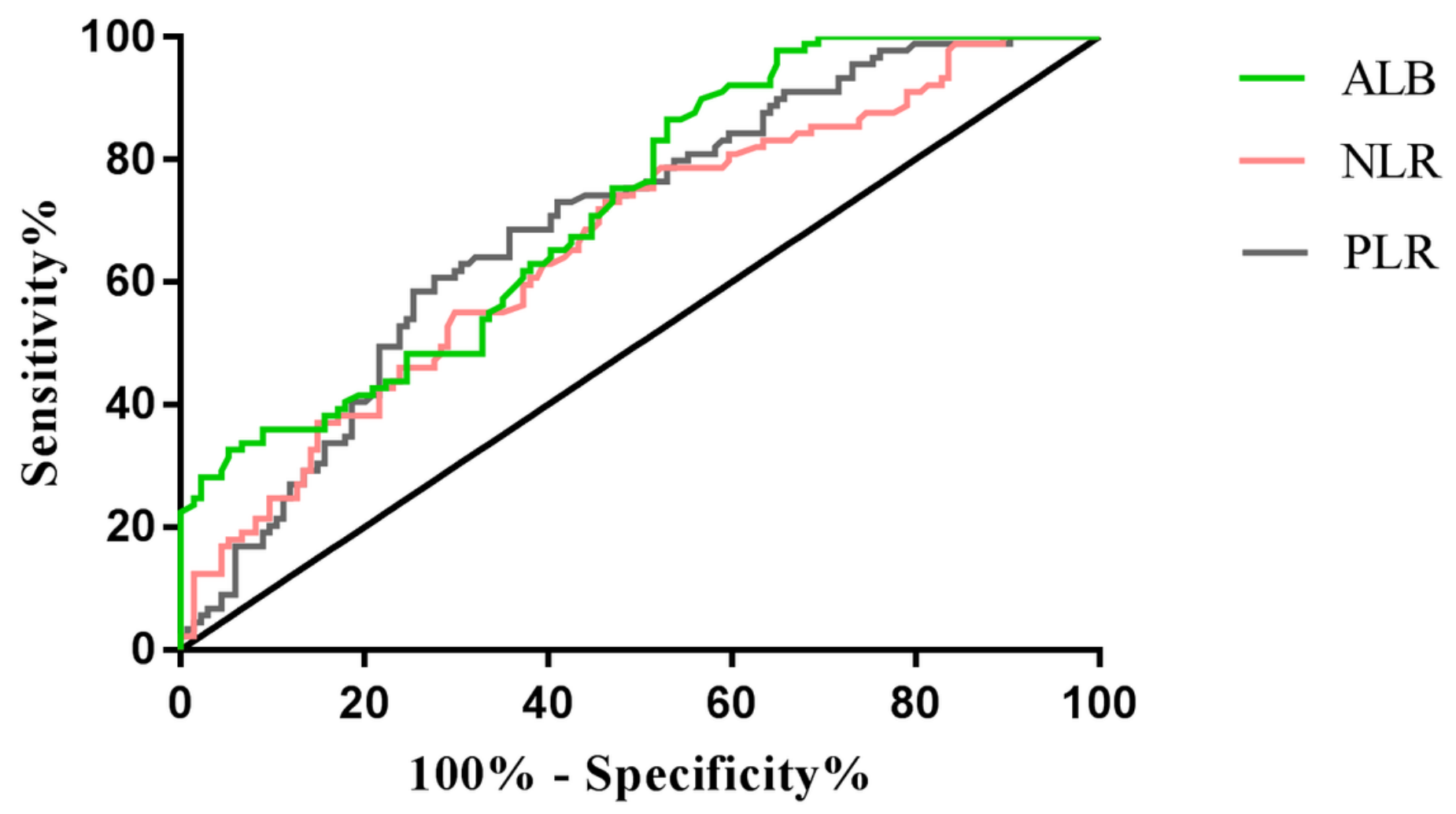

Figure 2

The ROC curve analysis was employed to calculate the truncation values AUCs, sensitivities, specificities, and $95 \%$ confidence intervals (Cls) of NLR, ALB, and PLR (Table 3 ), and the ROC curves of NLR, ALB, and PLR were are depicted in Figure 2.

\section{Supplementary Files}

This is a list of supplementary files associated with this preprint. Click to download.

- OnlineTable1.png

- OnlineTable2.png

- OnlineTable3.png

- OnlineTable4.png 